\title{
Actitudes de los docentes hacia la inclusión escolar de niños con autismo
}

\section{Attitudes of teachers towards the school inclusion of children with autism}

\author{
Rosa María Zambrano Garcés ${ }^{1 *}$ y Maria Daniela Orellana Zambrano ${ }^{2}$ \\ ${ }^{1}$ Universidad Católica de Cuenca, Extensión San Pablo de La Troncal \\ 2 Pontificia Universidad Católica de Quito \\ *rmzambranog@ucacue.edu.ec
}

DOI: https://doi.org/10.26871/killkana_social.v2i4.296

\begin{abstract}
Resumen
Ecuador ha expedido leyes y reglamentos en búsqueda de la aceptación de la diversidad dentro de la educación básica; sin embargo, más allá de los cambios estructurales y de legislación, todo proceso de educación inclusiva necesita implementar prácticas docentes que faciliten el potencial de aprendizaje de las personas con discapacidad. Este estudio, enmarcado en la línea de investigación equidad y educación, se planteó como objetivo analizar las actitudes de los docentes hacia la inclusión escolar de niños con autismo en la Unidad Educativa Particular en el Cantón La Troncal, Provincia Cañar, Ecuador. Participaron voluntariamente, ocho profesores de Educación General Básica, quienes tenían en su aula, por lo menos un niño con autismo. La metodología fue cualitativa con alcance descriptivo; se aplicó entrevista con preguntas abiertas considerando los siguientes ejes: La experiencia de los docentes, las características de los niños con autismo, tiempo y recursos de apoyo, formación docente y capacitación. Se llegó a la conclusión que la actitud del docente es trascendental durante el proceso de inclusión escolar; por lo tanto el análisis de sus percepciones, actitudes y expectativas son relevantes para el desarrollo exitoso de la educación inclusiva de los niños con autismo. Este estudio es un inicio para promover una cultura, políticas y prácticas inclusivas que provean a los docentes de recursos y apoyos específicos para que atiendan a los niños con autismo de una manera eficiente.
\end{abstract}

Palabras clave: Actitudes, docentes, inclusión, niños, autismo.

\begin{abstract}
Ecuador has issued laws and regulations in search of acceptance of diversity within basic education; however, beyond the structural changes and legislation, any inclusive education process needs to implement teaching practices that facilitate the learning potential of people with disabilities. This study, framed in the equity and education research line, aimed to analyze the attitudes of teachers towards school inclusion of children with autism at Unidad Educativa Particular, Cantón La Troncal, Provincia Cañar, Ecuador. Eight teachers of Basic General Education participated voluntarily, who had at least one child with autism in their classroom. The methodology was qualitative with descriptive scope; Interview with open questions was applied considering the following axes: The experience of teachers, the characteristics of children with autism, time and resources of support, teacher training and training. It was concluded that the attitude of the teacher is transcendental during the process of school inclusion; therefore, the analysis of their perceptions, attitudes and expectations are relevant for the successful development of inclusive education for children with autism. This study is a beginning to promote an inclusive culture, policies and practices that provide teachers with specific resources and supports to assist children with autism in an efficient manner.
\end{abstract}

Key words: Attitudes, teachers, inclusion, children, autism.

\section{Introducción}

Esta investigación surge porque en la Unidad Educativa Particular ubicada en el Cantón La Troncal, varios padres de familia manifestaban inconformidad a la autoridad institucional, con respecto a la actitud de los docentes hacia sus hijos diagnosticados con autismo, consideraban que era indispensable que dichos docentes reciban capacita- ción especializada para atender las necesidades educativas especiales de los niños. Es así como se presenta la pregunta ¿Cuáles son las actitudes de los docentes hacia la inclusión escolar de niños con autismo?

Desde el marco conceptual el estudio se fundamenta en las políticas y normativas de la Convención Internacional de los derechos de las personas con discapacidad (CIDPD), 
la Constitución de la República del Ecuador, la Ley Orgánica de Discapacidades y la Ley Orgánica de Educación Intercultural (LOEI).

Se profundiza en las concepciones teóricas de la inclusión escolar y las diferencias en las actitudes de los docentes hacia los niños con autismo que están influidas por la experiencia docente, las características de los niños con autismo, el tiempo que los docentes llevan en su aula con un niño con autismo, recursos de apoyo y formación docente y capacitación.

La investigación determina que durante los últimos años el sistema educativo ha atravesado por un profundo proceso de transformación con miras a la inclusión dentro de las aulas para lograr una educación de calidad y calidez para todos los niños sin exclusión alguna. Sin embargo, más allá de los cambios estructurales y de legislación, este estudio demuestra que todo proceso de educación inclusiva necesita implementar prácticas docentes que faciliten el potencial de aprendizaje de los niños con autismo. La información obtenida sirve de apoyo y orientación a los organismos de acreditación y aseguramiento de la calidad de la educación general básica para la articulación de lineamientos, estrategias y acciones que garanticen que los docentes eduquen de una manera inclusiva.

\section{Política y Normatividad}

La Convención Internacional de los derechos de las personas con discapacidad (CIDPD) en el artículo 24 establece que estas personas tienen derecho a la educación (Organización de las Naciones Unidas, 2006). Según Alcaín y Medina (2017) para que esta norma sea efectiva, sin discriminación y sobre la base de la igualdad de oportunidades, los estados que la han ratificado, asegurarán un sistema de educación inclusivo a todos los niveles.

Ecuador ratificó la Convención Internacional de los derechos de las personas con discapacidad en el año 2008; la educación inclusiva ecuatoriana está regentada por una amplia normativa legal, comenzando por la Constitución de la República (2008, p.36), que en su artículo 47 establece: "El Estado garantizará políticas de prevención de las discapacidades y, de manera conjunta con la sociedad y la familia, procurará la equiparación de oportunidades para las personas con discapacidad y su integración social".

La Ley Orgánica de Discapacidades (2012, p.11) plantea en el artículo 27:

Derecho a la educación.- El Estado procurará que las personas con discapacidad puedan acceder, permanecer y culminar, dentro del Sistema Nacional de Educación y del Sistema de Educación Superior, sus estudios, para obtener educación, formación y/o capacitación, asistiendo a clases en un establecimiento educativo especializado o en un establecimiento de educación escolarizada, según el caso.

La Ley Orgánica de Educación Intercultural (2011, p. 40) establece en el artículo 47: "El estado ecuatoriano garantizará la inclusión e integración de las personas con discapacidad en los establecimientos educativos, eliminando las barreras de su aprendizaje".

Históricamente en el Ecuador se establecieron dos sistemas de educación separados: educación regular para los niños o adolescentes que se consideraron "normales" y educación especial para los estudiantes con discapacidad. Actualmente la educación inclusiva rompe con este paradigma y busca un trabajo en conjunto de estos dos sistemas para atender a la diversidad. El Ministerio de Educación de Ecuador ha implementado 140 Unidades Distritales de Apoyo a la Inclusión (UDAI) a nivel nacional, con el objetivo de fortalecer la Educación Especializada e Inclusiva, y así garantizar el acceso, permanencia, participación y aprendizaje de los niños, niñas y jóvenes con necesidades educativas especiales asociadas o no a la discapacidad. Las Unidades de Apoyo a la Inclusión son las instancias técnico-operativas, que proporcionan apoyo técnico, metodológico y conceptual mediante el trabajo de un equipo de profesionales. Su misión es facilitar la inclusión de los niños, niñas y adolescentes con necesidades educativas especiales asociadas o no a una discapacidad, con preferencia de atención a quienes se encuentran en situación o riesgo de exclusión, marginación o de abandono del proceso educativo a través de tres ejes de atención: evaluación /ubicación, intervención, y seguimiento, favoreciendo su acceso, permanencia, participación, aprendizaje, promoción y culminación en el Sistema Educativo Nacional. Los apoyos están orientados al desarrollo de las instituciones mediante el énfasis en la disminución o eliminación de las barreras para el aprendizaje y la participación que se generan en los contextos.

\section{Referentes teóricos}

\subsection{Inclusión}

La inclusión es un movimiento mundial que pretende que los sujetos excluidos de los servicios básicos que presta un estado sean reconocidos como personas con derechos. De acuerdo a Booth y Ainscow (2002) la inclusión es un conjunto de procesos orientados a eliminar o minimizar las barreras que limitan el aprendizaje y la participación de todos los estudiantes.

El éxito de la educación inclusiva depende de ciertos aspectos relevantes que se relacionan según Eisenman, Pleet, Wandry y McGinley (2011), con el liderazgo de las instituciones educativas, una cultura colaborativa, arreglos y adaptaciones de infraestructura que facilitan el acceso, la posibilidad de compartir la experiencia de otros profesores y con el desarrollo profesional de especialistas.

Como señala Juan Felipe Carrillo (2014) la educación inclusiva está dirigida a la búsqueda de sociedades incluyentes y filosofía de la inclusión en materia de procesos educativos, considerándose tres aspectos fundamentales: potenciar y valorar la diversidad, promover el respeto a ser diferente y facilitar la participación de la comunidad en el marco de una estructura intercultural. Entonces, se puede afirmar que la educación inclusiva es un paradigma que 
se sale exclusivamente de lo académico y curricular para enfocarse en el aspecto social.

\subsection{Autismo}

Los niños con Trastorno del Espectro Autista, el cual es un trastorno del desarrollo con bases neurológicas que se caracteriza por deficiencias en las áreas de lenguaje, comunicación, expresión de afectos, interacción social y que exhibe conductas motoras repetitivas (DSM-5, 2013), no son fácilmente incluidos en las escuelas regulares por las barreras del medio ambiente y de las manifestaciones biológicas, cognitivas y conductuales que presentan estos niños.

Eugenio Bleuler (1906) fue quien introdujo el término autismo en el ámbito de la psiquiatría para describir los síntomas de pacientes adultos esquizofrénicos, entre los cuales se mencionan el retraimiento o desapego de la realidad; el absoluto o parcial predominio del "mundo interior"; pérdida del sentido de la realidad; y, pensamiento esquizofrénico (Frith, 1991; Paluszny, 1991; Polaino-Lorente, 1982).

Leo Kanner en 1943 identificó varios síntomas a los que denominó "autismo infantil precoz". Haciendo referencia a L.Wing (1982) los rasgos que Kanner consideró de importancia en el autismo fueron: falta de contacto afectivo con otras personas; insistencia obsesiva en mantener el ambiente sin cambios, a repetir una gama limitada de actividades ritualizadas, actividades de juego repetitivas y estereotipadas, escasamente flexibles y poco imaginativas; fascinación por objetos susceptibles de ser manipulados a través de finos movimientos motores; aparición de un tipo de lenguaje cuya función no parece ser la comunicación interpersonal, existencia de retraso y alteraciones en la adquisición y el uso del habla y el lenguaje, tendencia al empleo de un lenguaje no comunicativo y con alteraciones peculiares, como la ecolalia y la propensión a invertir los pronombres personales y; aparente fisonomía inteligente y pensativa con un alto potencial cognitivo que se manifiesta entre aquellos que saben hablar por medio de proezas de memoria, y entre aquellos que no hablan por sus buenos resultados en pruebas de ejecución.

En un comienzo el estudio del autismo estuvo influenciado por el psicoanálisis, y se consideraba a la familia como un factor determinante en el origen del mismo; progenitores, especialmente las madres, que no brindan el afecto necesario a sus hijos provocando el trastorno. Posteriormente las investigaciones determinaron indicios de una relación genética y neurobiológica con el autismo, y su génesis pasó del área emocional al área cognitiva. Algunos autores atribuyen a factores psicogenéticos (o ambientales-emocionales) como los posibles responsables; por el otro, se dice que los responsables son factores de tipo biogenéticos u organicistas, como la deficiencia en neurotransmisores, factores genéticos y virales (Baron-Cohen y P, 1998; Frith, 1991; Garanto, 1990; Paluszny, 1991; Wing, 1982).
El autismo es estudiado por científicos y profesionales que se sienten atraídos por las relaciones afectivas extrañas que los niños manifiestan. Se destaca que pese a todos los avances de la ciencia, todavía no se conocen los factores responsables de este trastorno mental que perturba al propio niño y a los familiares más allegados como son los padres. El autismo suele detectarse dentro de los tres primeros años de vida y existen diferentes grados de intensidad en una o más áreas con dificultades, teniendo así el diagnóstico un amplio espectro, desde patología severa, hasta el autismo de alto funcionamiento o síndrome de Asperger (Bettelheim, 2001).

Algunos niños mantienen un buen nivel académico, pero no pueden interactuar con sus pares, presentan problemas emocionales, de adaptación social y de conducta; otros niños con autismo pueden manifestar dificultades de aprendizaje y poca atención, conductas obsesivas y repetitivas, por lo que la inclusión suele ser más complicada para estos niños en comparación con los otros niños que tienen Necesidades Educativas Especiales.

\subsection{Actitudes docentes}

Etimológicamente actitud viene del latín aptitudini, que significa postura. La actitud es una posición u orientación el pensamiento, que se traduce en una forma determinada de pensar, actuar o reaccionar; es decir, la actitud viene a ser una forma de comportamiento de la persona frente a determinada circunstancia.

De acuerdo a Beltrán (1998) la actitud es una posición $\mathrm{u}$ orientación del pensamiento, que se traduce en una forma determinada de pensar, actuar o reaccionar. La actitud se compone de tres dimensiones: a) la cognoscitiva, formada por percepciones, creencias e información que se tiene sobre algo. b) La afectiva que implica sentimientos a favor o en contra de algo y c) la conductual referida a la tendencia a reaccionar hacia algo de una cierta manera.

Como parte fundamental del proceso de transformación educativa y social, se encuentra el rol del maestro y su actitud frente a la educación inclusiva; el docente es quien se convierte en el mediador de los procesos y portador de instrumentos que permiten al niño con autismo hacer frente a las diversas situaciones que se presentan día a día dentro del aula de clase. El docente es definido como agente clave para la construcción de una escuela inclusiva de calidad al ser el instrumento pedagógico por excelencia (Jordán, 2007). La herramienta más potente para el cambio es desde el profesorado y con el profesorado (Palomares, 2011).

La actitud del docente es determinante en la aplicación de políticas inclusivas, la revisión de la literatura establece que la gran mayoría de los profesores apoyan la inclusión; sin embargo, cuando están frente a un caso de niño con autismo, este entusiasmo va disminuyendo. Avramidis y Kalyva (2007) manifiestan que los docentes afirman tener actitudes positivas hacia la inclusión, aunque prefieren la presencia en el aula de un profesor de educación especial o 
que los niños con necesidades educativas especiales realicen sus estudios en aulas de educación especial.

\section{Referentes contextuales}

El análisis de percepciones, actitudes y expectativas del profesorado es crítico para el desarrollo exitoso de una política educativa inclusiva. Muchos estudios realizados en torno a las representaciones, creencias y actitudes de los profesores frente a la integración escolar, dan cuenta de que aquello que el profesor piensa sobre integración, se verá reflejado en la puesta en marcha que hace de la misma (Parrilla, 1992) . Las representaciones que tienen los individuos sobre determinado hecho, objeto o persona, en este caso el profesorado que trabaja con niños que tienen discapacidad en el aula común, traspasa la actuación pedagógica, dejando entrever lo que piensan o imaginan sobre estos niños. (Damm, 2009) .

Estudios de las percepciones y actitudes de los maestros/profesores hacia la inclusión de los niños con autismo se han realizado de una forma global, sin considerar ni prestar suficiente atención a las condiciones en que éstas se están llevando a cabo en los centros educativos (Chinner, 2011). Una de las barreras que los niños con autismo enfrentan y puede afectar su acceso, permanencia y participación dentro del sistema escolar, es la formación especializada del docente; la presencia de ella influye en actitudes positivas del docente hacia la inclusión (Rose, 2001; Idol, 2006; Horne y Timmons, 2009) ; la ausencia de la misma puede generar sensaciones de incompetencia, desinterés y rechazo (Sales, Moliner, y Sanchiz, 2001; Sánchez, Díaz, Sanhueza, y Fritz, 2008; Monereo, 2010).

Los estudios sobre educación inclusiva en Ecuador son escasos, por lo que se considera relevante conocer las diversas actitudes que pueden desarrollar los docentes hacia la inclusión escolar de los niños diagnosticados con autismo.

\section{Metodología}

\section{Participantes}

La presente investigación consta de 8 docentes que dictan clases de Primero a Séptimo Año de Educación Básica:

- $\quad$ Docente de Lengua y Literatura, sexo femenino, 35 años edad, Licenciada en Educación, Mención Lengua y Literatura.

- Docente de Matemática, sexo masculino, 28 años de edad, Ingeniero en Contabilidad y Auditoría.

- Docente de Ciencias Naturales, sexo masculino, 40 años de edad, Licenciado en Educación.

- Docente de Estudios Sociales, sexo femenino, 29 años de edad, Abogada.

- Docente de Educación Estética, sexo femenino, 42 años de edad, Licenciada en Educación.

- Docente de Educación Física, sexo masculino, 39 años de edad, Licenciado en Educación Inicial y Parvularia.
- Docente de Lengua Extranjera, sexo femenino, 38 años de edad, Licenciada en Educación Mención Idioma Inglés.

- Docente de Proyectos Escolares, sexo femenino, 30 años de edad, Ingeniera en Administración de Empresas.

El estudio fue realizado en la Unidad Educativa Particular en el Cantón La Troncal, Provincia Cañar, Ecuador, durante el año 2016.

\section{Materiales}

Se utilizó la entrevista a los docentes a base de preguntas abiertas como instrumento para recoger la información por permitir profundizar de forma cualitativa en las representaciones de docentes de niños diagnosticados con trastorno autista del desarrollo. Para realizar la entrevista se realizó un listado temático para orientar el trabajo y profundizar en los siguientes ejes:

1) La experiencia de los docentes.

2) Las características de los niños con autismo.

3) Tiempo y recursos de apoyo.

4) Formación docente y capacitación.

Se aplicó una ficha de datos donde se recopiló la edad, sexo, grado de instrucción, años de experiencia como docente.

\section{Procedimiento}

Con el consentimiento informado de los docentes de la unidad educativa se realizó la entrevista a los docentes en el Departamento de Consejería Estudiantil; se transcribieron las respuestas y se agrupó la información para el análisis de contenido.

La interpretación y análisis de la información se realizó mediante el contraste de la investigación bibliográfica y los resultados obtenidos de los docentes, en base a los cuales se sustentan las conclusiones.

\section{Diseño}

En esta investigación de diseño no experimental de enfoque cualitativo con alcance descriptivo y exploratorio se utilizó el estudio de casos lo que permitió recoger, interpretar y analizar los datos sobre las percepciones, opiniones, actitudes, sentimientos o conductas de los docentes que dictan clases a los niños diagnosticados con autismo.

\section{Resultados y Discusión}

\subsection{Resultados}

Se analizaron las respuestas de ocho docentes que dictan las siguientes asignaturas: Lengua y Literatura, Matemática, Ciencias Naturales, Estudios Sociales, Educación Estética, Educación Física, Lengua Extranjera y Proyectos Escolares en cada uno de los años de Educación General Básica.

La experiencia de los docentes. Se entiende por experiencia docente la práctica educativa que poseen los profesores y el tiempo de ejercicio profesional. La mayoría de los docentes no ha tenido una experiencia previa con niños del Trastorno del Espectro Autista, experimentan ansiedad 
y temores por una situación completamente nueva; aquellos docentes que si han tenido la oportunidad de hacerlo, manifiestan una actitud positiva hacia la inclusión.

\section{Experiencia de los Docentes}

- “... Soy docente desde hace ocho años y honestamente es la primera vez que tengo un niño que manifiesta conductas extrañas y difíciles de controlar, no sé cómo hacer, estoy atemorizado,...."

- “....desde hace diez años que soy docente y es la primera vez que trabajo con un niño diagnosticado con Trastorno del Espectro Autista, no sé cómo hacer y estoy ansioso..."

- “...Trabajo como docente desde hace doce años y no sé lo que es el autismo, no me siento preparado para trabajar con niños autistas...."

- “... Soy docente de la vieja guardia y es un trastorno nuevo para mí, las conductas repetitivas me asus$\tan . . . . "$

- “....En mis doce años de docencia, esto es nuevo para mí y muy difícil, el niño se bota al suelo y no sé cómo controlarle...."

- “....Soy docente desde hace tres años y he tenido la bendición de trabajar en dos oportunidades con niños con Trastornos del Espectro Autista, aprendí que cada uno es un mundo singular y hay que realizar las adecuaciones curriculares...."

- “...Laboro en esta Unidad Educativa desde hace dos años y el año pasado tuve la oportunidad de trabajar con un niño con Síndrome de Asperger, sé que no es lo mismo pero hay ciertas conductas que son muy parecidas; lo importante es trabajar en equipo con la psicopedagoga, los padres y el niño....."

- “....Trabajo como docente desde hace cuatro años y es un reto para mí cada ocasión que tengo un niño con este trastorno, es una oportunidad de aprendizaje para mí...."

Las características de los niños con autismo. El Trastorno del Espectro Autista genera rechazo en la mayoría de los docentes entrevistados debido a que los niños presentan dificultades en la interacción y relaciones sociales, comunicación, imaginación y rigidez mental, dificultades sensoriales y motoras, dificultades emocionales y destrezas en el trabajo.

\section{En referencia a las relaciones sociales}

- “.... NN intenta participar y hacer amigos, pero parece que solo consigue sacar de quicio a los demás. Nadie quiere estar con él...."

- “.... NN en el recreo lo único que hace es pasar solo. En clase no soporta ser el centro de atención...."

- “.... NN no diferencia cuando los demás le hacen una broma y cuándo no...."

- “... NN se llena de iras, hace rabietas y da golpes contra la pared...."

- “... NN interrumpe la clase todo el tiempo...."

\section{En referencia a la comunicación}

- “.... Cuando se le da una instrucción a NN, parece que si escucha pero luego no logra hacer la tarea..."

- “... NN parece inteligente pero cuando explico algo nuevo parece no enterarse de nada...."

- “... NN interpreta todo al pie de la letra...."

- “.... NN es rígido y monótono en su hablar, no tiene expresión facial alguna..."

- “... NN habla demasiado alto..."

- “... NN hace la misma pregunta una y otra vez...."

\section{En referencia a la imaginación y rigidez mental}

- “.... Es muy difícil hablar con NN, le gusta hablar solamente de fechas de nacimiento...."

- “.... A NN le asustan los pájaros, no quiere salir al recreo por temor a encontrar uno...."

- “.... A NN le gusta que todo se haga siguiendo un orden estricto...., si le interrumpo la rutina, se enoja mucho"

En referencia a las dificultades sensoriales y motoras

- “... Cuando hay mucha gente, NN sale corriendo...."

- “.... Cuando NN entra al bar de la escuela donde hay mucho ruido, se tapa los oídos y empieza a balancearse, como si se fuera a caer..."

- “.... Si alguien le topa alguna parte de su cuerpo, NN grita...."

En referencia a las dificultades emocionales

- “.... NN se llena de iras sin razón..."

- “... NN se frustra cuando se hacen cambio de planes...."

- “.... NN comienza a darse cuenta que es diferente a los demás, y pregunta ¿por qué? ....."

- “.... Hay ocasiones en que noto a NN sin ánimos de hacer las cosas...."

\section{En referencia a las destrezas de trabajo}

- “.... Le pido a NN que trabaje sobre las partes de una planta, pero se niega hacerlo, prefiere trabajar sobre las direcciones de las casas de los tíos...."

- “.... NN nunca tiene los materiales a mano para realizar las tareas..."

- “....NN siempre está deambulando durante la clase y no se sienta a hacer la tarea...."

- “.... Siempre hace falta que esté a lado de NN para que haga la tarea...."

- “.....NN tiene dificultades para escribir...."

\section{Tiempo y recursos de apoyo}

Tiempo y recursos de apoyo: Se entiende por tiempo la disponibilidad del docente para planificar y coordinar las actividades curriculares para cada niño con necesidad educativa especial; y los recursos de apoyo tanto humanos como materiales para desarrollar su clase de acuerdo a la adaptación curricular correspondiente. La mayoría de los docentes manifestaron no contar con el tiempo suficiente para planificar sus actividades pedagógicas para cada niño con Trastorno del Espectro del Autismo. En lo que respecta a los recursos de apoyo indicaron que cuenta con todo 
el respaldo de la psicopedagoga terapéutica de la Unidad Educativa así como de los padres de familia; y en cuanto a los recursos materiales los docentes refirieron que tienen dificultades para realizar las adaptaciones curriculares y aplicar las estrategias de enseñanza-aprendizaje puesto que no siempre hay el material didáctico adecuado para dictar clases con niños con autismo.

- “....Se me complica la clase cuando tengo un niño con autismo, debo realizar las adaptaciones curriculares y a veces no tengo suficiente tiempo...."

- “....Yo quisiera que niños como los autistas vayan a escuelas especiales...."

- “.... No es mucho lo que yo puedo hacer con un niño con autismo, no me corresponde a mi hacer las adaptaciones curriculares, para eso está la psicopedagoga terapéutica...."

- “.... La Unidad Educativa no cuenta con el material didáctico apropiado para niños con autismo...."

- “....Mi trabajo es dictar la clase para todos, los padres deben preocuparse de brindar apoyo sicopedagógico en la tarde...."

- “....Es difícil cuando hay que trabajar con niños con autismo, no sé cómo hacer con ellos...."

- “....Considero que cuento con el tiempo suficiente para hacer la planificación curricular adaptada a las necesidades de los niños con autismo..."

- “.... La Unidad Educativa nos proporciona todo el apoyo académico y material didáctico para trabajar con niños con autismo...."

\section{Formación docente y capacitación}

Formación Docente y Capacitación. Es necesario que los docentes tengan formación profesional que les proporcione las herramientas adecuadas para responder a las necesidades educativas especiales de un niño con autismo. Así mismo, es recomendable contar con capacitación continua que les permita actualizarse para atender las demandas emergentes de los estudiantes en el proceso de enseñanza aprendizaje. En general los docentes entrevistados no se sienten competentes ni a gusto dictando clases a niños con el Trastorno del Espectro Autista, solo unos pocos docentes han sido capacitados durante su formación universitaria; aquellos que si han recibido capacitación poseen una actitud más positiva y aceptan la asistencia de la psicopedagoga terapéutica de la institución educativa.

- “....Se requiere de una capacitación adecuada, conocer qué mismo es el autismo y cuáles son los síntomas y signos del trastorno..."

- “....En la universidad no recuerdo haber sido capacitado para saber cómo hacer con los niños con Trastorno del Espectro Autista...."

- “...En realidad no he recibido capacitación alguna sobre cómo manejar los casos de autismo...."

- “.... Si bien es cierto en la universidad nos enseñan sobre el Trastorno del Espectro Autista, se me hace difícil realizar las adaptaciones curriculares...."
- “....Una cosa es conocer sobre el Trastorno del Espectro Autista y otra cosa es la práctica....."

- “.....En mi formación universitaria estudié algo sobre Autismo dentro de los Trastornos del Aprendizaje....." “.....Tengo los conocimientos teóricos sobre el Trastorno del Espectro Autista, y hago las adaptaciones curriculares de acuerdo a sus necesidades especiales...."

- “.... Constantemente me capacito en cómo ayudar a los niños con Necesidades Educativas Especiales, como son los niños con Trastorno del Espectro Autista, y busco el apoyo de la psicopedagoga terapéutica de la Unidad Educativa para que el niño sea parte del proceso enseñanza aprendizaje..."

\subsection{Discusión}

Larrivée y Cook (1978) hipotetizan que las actitudes de los maestros hacia la inclusión escolar están afectadas por diversas variables institucionales. Escogen por una parte variables fijas del entorno: nivel educativo, tamaño de la clase, tamaño de la escuela y tipo de emplazamiento de la escuela, y, por otra parte, variables perceptivas de los maestros: grado de éxito conseguido con los alumnos con necesidades especiales, nivel de apoyo administrativo recibido, disponibilidad de servicios de apoyo. Los resultados de la investigación de Larrivée y Cook (1978) son los siguientes:

1) Las actitudes hacia la inclusión de los maestros de clases normales tienden a ser más positivas a medida que el nivel educativo desciende.

2) Parece ser que las actitudes hacia la inclusión de los maestros de clases normales no se ven afectadas por variables como el tamaño del aula, tamaño de la escuela y tipo de escuela.

3) Se observa una gran relación entre las actitudes hacia la inclusión y la percepción del maestro del grado de éxito obtenido, el nivel de apoyo administrativo recibido y la disponibilidad de servicios de apoyo. Siendo la variable más importante que afecta a las actitudes hacia la inclusión la percepción por el profesor del éxito obtenido con este tipo de niños. La discusión de esta relación lleva los autores a postular que esta percepción por el profesor del éxito conseguido está en función de muchas variables, tales como nivel de información, conocimiento alcanzado, adquisición de habilidades específicas, contacto y experiencia con los niños excepcionales y de las actitudes: aspectos que no están claros y que exigirían estudios adicionales.

Los docentes en su práctica profesional no sólo deben dominar los contenidos que imparten sino, que es necesario que faciliten el aprendizaje y la participación de todos los estudiantes, propiciando oportunidades de mayor desarrollo e inclusión educativa(Sola, 1997). Esta investigación demuestra que los siguientes factores: La experiencia de los docentes, las características de los niños con autismo, tiempo y recursos de apoyo, formación docente y capacitación influyen para que los docentes manifiesten una 
actitud positiva o negativa frente a la inclusión de niños con Trastorno del Espectro Autista.

El estudio establece que los años de experiencia de los docentes influyen en su actitud, quienes tienen menos años de experiencia dictando clases manifiestan una actitud más positiva que aquellos con más experiencia. De Boer \& Minnaert (2011) plantean que los profesores que tienen experiencias previas en educación inclusiva muestran una actitud más positiva que aquellos con menos experiencia en contextos inclusivos.

De esta manera se puede considerar que la experiencia de los docentes influye en dos direcciones. Por un lado, la cantidad de años de trabajo, más tiempo como docente no favorece a la inclusión educativa; por otro lado, la experiencia previa en educación inclusiva impacta positivamente en la actitud del docente frente a aquellos docentes que no han tenido la oportunidad de trabajar con niños con autismo.

Huang \& Diamond (2009), manifiestan que el tipo de discapacidad parece jugar un importante rol en la disposición de profesores y administrativos hacia la inclusión. El Trastorno del Espectro de Autismo abarca una serie de características como son dificultades de interacción social, dificultades en la comunicación y rigidez de pensamiento, dificultades emocionales, dificultades sensoriales y motoras, destrezas de trabajo poco desarrolladas; el presente estudio determina que en muchas de las ocasiones esto ha generado ansiedad en los maestros y sienten que no tienen la confianza necesaria como para ayudar a estos niños a desenvolverse en el proceso enseñanza aprendizaje.

Horne \& Timmons (2009) hacen notar que algunas de las preocupaciones de los profesores se relacionan con el tiempo disponible de planificación y el cubrir las necesidades de todos los estudiantes. Esta investigación indica por una parte, que el docente no cuenta con el tiempo suficiente para organizar su trabajo lo cual genera una actitud de rechazo hacia las adaptaciones curriculares. Por otra parte, la presencia de especialistas en inclusión como la psicopedagoga terapéutica y el psicólogo educativo es la mejor opción para los niños con Trastorno del Espectro Autista, pues son ellos quienes coordinan las adaptaciones curriculares con los docentes de la Unidad Educativa. Jordan, Glenn \& McGhie-Richmond (2010) indican que cuando las escuelas tienen acceso a una gran variedad de apoyos y a diversificadas estrategias de enseñanza, ellas pueden ser efectivas incluyendo a diversos estudiantes y manteniendo altos niveles de logros académicos.

En cuanto a la capacitación de los docentes para trabajar con niños con Trastorno del Espectro Autista, este estudio señala que los docentes se sienten poco competentes para enseñar en un ambiente inclusivo, sin embargo, aquellos docentes que si han recibido capacitación poseen una actitud más positiva hacia la educación inclusiva. En este mismo sentido, De Boer \& Minnaert (2011) manifiestan que los profesores/as no se autocalifican como bien preparados para incluir estudiantes con discapacidad en sus aulas.
Horne \& Timmons (2009) enfatizan la importancia de contar con oportunidades de desarrollo profesional continuo, para así responder efectivamente al creciente número de necesidades especiales que presentan los estudiantes en la sala de clases, ya que establecen que los profesores se sienten insuficientemente preparados para cubrir las necesidades de sus estudiantes con Necesidades Educativas Especiales.

\section{Conclusiones}

A los niños con autismo hay que considerarles desde su singularidad; ingresan a la escuela para aprender, según sus necesidades y de acuerdo con sus posibilidades para construir conocimientos en la interacción social con sus pares y con el docente.

La tarea del profesional que recibe niños diagnosticados con autismo consiste en capacitarse para convertirse en un asesor y consultor de los diferentes aspectos de la vida del niño y su familia. De manera adicional, trabajar en equipos conformados por docentes, terapeutas, médicos o cualquier profesión afín, con el objetivo de establecer una comunicación integral entre los miembros; lo que conduce a un enriquecimiento constante, potenciación recíproca, competencia sana, superación sobre la base de resolución de conflictos.

La introducción en las escuelas de políticas educativas inclusivas significa cambios importantes en los roles y responsabilidades de los profesionales de la educación. Estos cambios generalmente no se han visto acompañados de un análisis previo de su pensamiento, creencias y actitudes hacia este proceso, cosa que puede estar comprometiendo seriamente el desarrollo de la inclusión de los niños con autismo. Dado que la investigación sobre el pensamiento del docente demuestra que las percepciones y actitudes definen los procesos de pensamiento, acción y disposición al cambio del profesorado ante determinados retos, el estudio de sus actitudes se convierte en elemento determinante para conocer el desarrollo y comprender mejor el comportamiento instructivo del docente en el aula.

Asumir la diversidad, desde sus diferentes dimensiones, como aquellas de tipo cultural, de capacidades, motivaciones y expectativas, desde los más a menos dotados, como es el caso de los niños con autismo, implica un gran reto, debido al conjunto de creencias, representaciones y estereotipos que maneje la sociedad, de las personas con autismo, y, por consiguiente, con las concepciones que se va formando también el profesorado.

\section{Referencias Bibliográficas}

Alcaín-Martínez, E., y Medina-García, M. (2017). Hacia una educación universitaria inclusiva: realidad y retos. Revista Digital de Investigación en Docencia Universitaria, 11(1), 4-19. Descargado 20 de septiembre de 2018, de http://dx.doi.org/ $10.19083 /$ ridu.11.530 
Asamblea Nacional República del Ecuador. (2008). Constitución de la república del ecuador. Descargado 24 de septiembre de 2018, de https: / / goo.gl/ Wfz8z3 (RO 449)

Asamblea Nacional República del Ecuador. (2011). Ley orgánica de educación intercultural. Descargado 17 de octubre de 2018, de https://goo.gl/Efbna 6 (RO 417)

Asamblea Nacional República del Ecuador. (2012). Ley orgánica de discapacidades. Descargado 23 de septiembre de 2018, de https: / / goo.gl / JCV2gV (RO 796)

Avramidis, E., y Kalyva, E. (2007). The influence of teaching experience and professional develpment on greek teachers'attitudes towards inclusion. European Journal of Special Needs Education, 22, 367-289.

Baron-Cohen, S., y P, B. (1998). Autismo: una guía para padres. Madrid: Alianza Editorial, S.A.

Beltrán, J. (1998). Procesos, estrategias y técnicas de aprendizaje. Madrid: Ed. Síntesis.

Bettelheim, B. (2001). La fortaleza vacía: autismo infantil y el nacimiento del yo. Barcelona: Paidós.

Bleuler, E. (1906). Dementia praecox or the group of schizophrenics. Nueva York: International Universities Press.

Carrillo, J. (2014). La educación inclusiva en educación superior. Descargado 22 de septiembre de 2018, de https://goo.gl/YTpjqu (Video)

Chinner, E. (2011). Las percepciones y actitudes del profesorado hacia la inclusión del alumnado con necesidades educativas especiales como indicadores del uso de prácticas educativas inclusivas en el aula. Universidad de Alicante. (Tesis doctoral)

Damm, X. (2009). Representaciones y actitudes del profesorado frente a la integración de niños/as con necesidades educativas especiales al aula común. $R e$ vista latinoamericana de educación inclusiva, 3(1), 25-35.

De Boer, y Minnaert, A. (2011). Regular primary schoolteachers' attitudes towards inclusive education: A review of the literature. International Journal of Inclusive Education, 15, 331-353.

Eisenman, L. T., Pleet, A. M., Wandry, D., y Mcginley, V. (2011). Voices of special education teachers in an inclusive high school: Redefining responsibilities. Remedial and Special Education, 32, 91-104.

Frith, U. (1991). Autismo. hacia una explicación del enigma. México: Editorial Alianza.

Garanto, J. (1990). El autismo. aproximación nosográficadescriptiva y apuntes psicopedagógicos. Barcelona: Editorial Harder.

Horne, P. E., y Timmons, V. (2009). Making it work: Teachers' perspectives on inclusion (Vol. 13).

Huang, H., y Diamond, K. E. (2009). Early childhood teachers' ideas about including children with disabilities in programmes designed for typically developing children (Vol. 56).

Idol, L. (2006). Toward inclusion of special education students in general education. a program evaluation of eight schools. Remedial and Special Education, 27(2), 77-94.

Jordan, A., Glenn, C., y Mcghie-Richmond, D. (2010). The supporting effective teaching (set) project: The relationship of inclusive teaching practices to teachers' beliefs about disability and ability, and about their roles as teachers (Vol. 26).

Jordán, J. A. (2007). Educar en la convivencia en contextos multiculturales. En Educación para la convivencia intercultural (p. 59-94). Madrid: La Muralla.

Larrivée, B., y Cook, L. (1978). The effect of institutional variables on teacher's attitude toward mainstreaming. Toronto, Canadá. (Paper presented at the meeting of the American Educational Research Association)

Monereo, C. (2010). La formación del profesorado: una pauta para el análisis e intervención a través de incidentes críticos (Vol. 52). 149-178.

Organización de las Naciones Unidas. (2006). Convención sobre los derechos de las personas con discapacidad. Descargado 23 de septiembre de 2018, de https://goo.gl/26b9oG

Palomares, A. (2011). El modelo docente universitario y el uso de nuevas metodologías en la enseñanza, aprendizaje y evaluación.

Paluszny, M. (1991). Autismo. guía práctica para padres y profesionales. México.

Parrilla, A. (1992). El profesor ante la integración escolar: investigación y formación. Buenos Aires: Cincel.

Polaino-Lorente, A. (1982). Introducción al estudio científico del autismo infantil. Madrid: Editorial Alhambra.

Rose, R. (2001). Primary school teacher perceptions of the conditions required to include pupils with special educational needs. Educational Review, 53(2), $147-$ 156.

Sales, A., Moliner, O., y Sanchiz, M. L. (2001). Actitudes hacia la atención a la diversidad en la formación inicial del profesorado. Revista Electrónica Interuniversitaria de Formación del profesorado, 4(2). Descargado de https: / / goo.gl/Wtht jQ

Sánchez, A., Díaz, C., Sanhueza, S., y Fritz, M. (2008). Percepciones y actitudes de los estudiantes de pedagogía hacia la inclusión educativa. Estudios pedagógicos, 34(2), 169-178.

Sola, T. (1997). La formación inicial y su incidencia en la educación especial. En A. Sánchez-Palomino y J. Torres-González (Eds.), Educación especial i. una perspectiva curricular, organizativa y profesional. Madrid: Pirámide.

Wing, L. (1982). Autismo infantil. aspectos médicos y educativos. Madrid: Editorial Santillana. 
Recibido: 27 de julio de 2018

Aceptado: 27 de noviembre de 2018

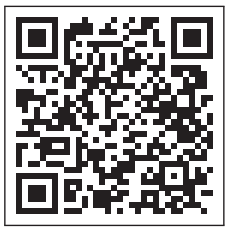


